\title{
Identificación de ácidos grasos y compuestos fenólicos de los aceites extraídos a partir de semillas de Ulex europaeus
}

\section{Identification of fatty acids and phenolic compounds of the oils extracted from seeds of Ulex europaeus}

\author{
Identificação de ácidos graxos e fenólicos dos óleos \\ extraídos de sementes de Ulex europaeus
}

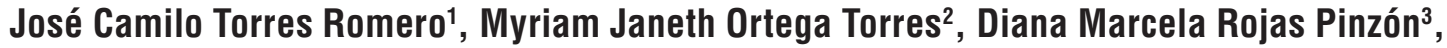
Jhon Infante-Betancour ${ }^{4}$ \& Leslie Yaneth Leal Mejía ${ }^{5}$

'Licenciado en Biología, Magister en Ciencias - Bioquímica, Doctor en Bioquímica. ²icenciada en Biología. Magister en Producción Animal- Genética Molecular Animal. Estudiante Doctorado en Producción Animal. Universidad Nacional de Colombia. Bogotá. Colombia. ${ }^{3}$ Estudiante Ingeniería Ambiental. Universidad Nacional Abierta y a Distancia UNAD. Bogotá. Colombia. ${ }^{4}$ Biólogo, Magister en Ciencias -Biología. ${ }^{5}$ Bióloga, Especialista en Docencia Universitaria, Magister en Ciencias -Biología.

1, 2, 3, 5 Escuela de Ciencias Agrícolas, Pecuarias y del Medio Ambiente -ECAPMA. Universidad Nacional Abierta y a Distancia UNAD. Bogotá. Colombia. ${ }^{4}$ Yoluka Ong Fundación de Investigación en Biodiversidad

y Conservación. Bogotá. Colombia.

1jose.torres@unad.edu.co, 2myriam.ortega@unad.edu.co, 32dianamarcelarojaspinzon@gmail.com, 4jhon.infante@yoluka.org.co, ${ }^{5}$ leslie.leal@unad.edu.co
\end{abstract}

\section{Resumen}

Ulex europaeus es una planta de origen mediterráneo, introducida en Colombia como cerca viva que ha adquirido gran importancia debido a su carácter invasor principalmente en las zonas de alta montaña. El conocimiento de su biología es una herramienta fundamental para la implementación de medidas de manejo que permitan su control y minimicen su impacto sobre los ecosistemas. El estudio de las moléculas y principios activos podría generar información valiosa a este respecto. La investigación reporta algunos ácidos grasos y compuestos fenólicos conseguidos de la extracción oleosa de las semillas e identificados por cromatografía de gases acoplada a espectrometría de masas. Dentro de los resultados destaca la presencia de ácido linoleico y también la presencia de tocoferoles que podrían ser útiles para aplicaciones alimentarias e industriales. Se concluye que la especie podría ser utilizada con fines industriales.

Palabras clave: ácido linoleico, cromatografía, tocoferoles, especie invasora.

\begin{abstract}
Ulex europaeus is a plant of Mediterranean origin, introduced in Colombia as a living fence that has acquired great importance due to its invasive character mainly in high mountain areas. Knowledge of their biology is a fundamental tool for the construction of management measures that allow their control and minimize their
\end{abstract}


impact on ecosystems. The study of molecules and active principles could generate valuable information in this regard. The work reports some fatty acids and phenolic compounds obtained from the oily extraction of the seeds and identified by gas chromatography coupled to mass spectrometry. The results highlight the presence of Linoleic acid and also the presence of Tocopherols that could be useful for food and industrial applications. It is concluded that the species could be used for industrial purposes.

Key-words: Linoleic acid, chromatography, Tocopherols, invasive species.

\section{Resumo}

Ulex europaeus é uma planta de origem mediterrânea, introduzida na Colômbia como uma cerca viva que adquiriu grande importância devido ao seu caráter invasivo principalmente em áreas de alta montanha. O conhecimento de sua biologia é uma ferramenta fundamental para a implementação de medidas de manejo que permitem seu controle e minimizem seu impacto nos ecossistemas. O estudo de moléculas e ingredientes ativos poderia gerar informações valiosas a esse respeito. A pesquisa relata alguns ácidos gordurosos e compostos fenólicos obtidos a partir da extração oleosa das sementes e identificados por cromatografia gasosa acoplada à espectrometria de massa. Os resultados destacam a presença de ácido linoleico e também a presença de tocoferóis que podem ser úteis para aplicações industriais e alimentares. Conclui-se que as espécies podem ser usadas para fins industriais.

Palavras chave: ácido linoléico, cromatografia, tocoferóis, espécies invasivas.

\section{Introducción}

Las especies invasoras han sido identificadas como uno de los principales motores de perdida de la biodiversidad del planeta (Genovesi \& Shine, 2004). En Colombia se han registrado 274 especies de plantas introducidas con antecedentes de invasión en el mundo (Cárdenas-López et al., 2010). Una de las especies que genera más alarmas en el país es Ulex europaeus Fabaceae una especie nativa de Europa (Kaufman, 2012), e introducida a Colombia en los años setenta para la construcción de cercas vivas en algunos páramos (Beltrán-G \& Barrera-Cataño, 2014; Guio et al., 2015) que esta categorizada a nivel nacional como una especie de alto riesgo de invasión (CárdenasLópez et al., 2010). Amaya \& Rengifo (2010) con base en la información de varios autores atribuyen su carácter invasivo a su forma de crecimiento a manera de matorral denso, su crecimiento perenne a largo plazo, la gran resistencia de su banco de semillas y su reproducción de tipo asexual.
En Colombia no se han realizado estudios de la fotoquímica de esta especie que puedan contribuir con información para generar medidas de manejo. En el mundo, las moléculas de esta especie han sido reportadas por presentar actividad con diversos efectos farmacológicos tales como hipolipemiante, hipoglicémico, hipotensor, antiinflamatorio, gastroprotector, hepatoprotector, antioxidante, antiosteoartrítico, antiasmático; entre otros, sin que ello se acompañe de toxicidad relevante (Rivera \& Obón, 1995; Spínola et al., 2016). Los principales compuestos que contiene -polifenoles, esteroles, glicósidosisotiocianatos y carbamatos- están en toda la planta, y presenta aceite en las semillas, constituido por ácidos grasos entre 8 a 26 átomos de carbono, además de compuestos fenólicos (Tighe-Neira et al., 2014). Esta planta se distribuye en Colombia en la región andina entre los 2000 y 3180 msnm (Bernal et al., 2016). Sin embargo, no se encontraron trabajos 
que describan los ácidos grasos provenientes, aceite obtenido de las semillas de dicha especie que crece en nuestro país, así como de los compuestos fenólicos. Teniendo en cuenta las posibles aplicaciones en las industrias farmacéuticas y alimentarias de $U$. europeaus y la necesidad de su control como especie invasora en el contexto colombiano, se procedió a determinar el contenido de ácidos grasos y compuestos fenólicos de dicho aceite por cromatografía gaseosa acoplada a espectrometría de masas CG-MS. Este artículo de investigación constituye una contribución al estudio químico de este aceite, pues permite profundizar en el conocimiento de sus componentes y visualizar posibles aplicaciones.

\section{Métodos}

\section{Recolección de Semillas}

Para la recolección de las muestras se procedió a recolectar semillas en los cerros orientales (04 31' 26,0" N / 074 05' 05,3') en la localidad de San Cristóbal de Bogotá, Colombia. La colecta fue manual tomando muestras aleatoriamente en un área de $20 \mathrm{~m}^{2}$ de invadida por retamo, $4 \mathrm{~kg}$ de semillas fueron embaladas en bolsas ziploc para su almacenamiento y posterior transporte al laboratorio.

\section{Preparación de la muestra}

Las muestras fueron preparadas por el laboratorio del Grupo de Investigación en Substancias Bioactivas (GISB) de la Universidad de Antioquia. Brevemente; para la obtención los ácidos grasos, se tomaron aproximadamente $50 \mathrm{mg}$ de muestra, se diluyeron en cloroformo y a una alícuota de $50 \mu \mathrm{L}$ se le evaporó el solvente, posteriormente, la muestra fue derivatizada con una solución de ácido sulfúrico y metanol, seguida de una micro extracción líquidolíquido con hexano. La fase orgánica resultante fue dispuesta para el análisis.
Para la determinación de compuestos fenólicos, $50 \mathrm{mg}$ del aceite fueron diluidos en cloroformo, posteriormente una alícuota de $50 \mu \mathrm{L}$ fue llevada a sequedad y se agregó reactivo derivatizante $\mathrm{N}, \mathrm{O}$-Bis(trimethylsilyl) trifluoroacetamide (BSTFA) dando un tiempo de reacción de 12 h, después del cual se dispuso la muestra para su análisis cromatográfico.

Determinación de ácidos grasos y compuestos fenólicos mediante GC/MS: Las muestras fueron analizadas por el laboratorio del Grupo de Investigación en Substancias Bioactivas (GISB) de la Universidad de Antioquia. En general, previo al análisis por GC-MS de las muestras de aceite, se aplicaron técnicas de derivatización utilizando agentes metilantes y sililantes para el tratamiento de los ácidos grasos y compuestos fenólicos, respectivamente. Posteriormente las sustancias de interés fueron separadas por cromatografía de gases y analizados por espectrometría de masas. Para la identificación de los compuestos, se realizó la comparación de los patrones de fragmentación de las sustancias detectadas con la base de datos espectral NIST tal como lo indica Marriott \& Shellie (2002).

\section{Resultados}

\section{Determinación de ácidos grasos en el aceite}

En las muestras analizadas se encontraron los picos correspondientes a los estándares de metil ésteres de ácidos grasos a diferentes tiempos de retención, identificando los siguientes: láurico, mirístico, palmítico, linoleico, oleico, eláidico, esteárico, cis-11-eicosanoico, eicosanoico y docosanoico (Tabla 1 y Figura 1). En la Tabla 1 se muestran los tiempos de retención y el porcentaje del ácido graso con respecto a la fracción oleica de la semilla y en la Figura 1 se muestra el perfil cromatográfico de los ácidos grasos presentes en la muestra. 
Tabla 1. Ácidos grasos presentes en aceite de semillas de Ulex europaeus.

\begin{tabular}{l|c|c}
\hline \multicolumn{1}{c}{ Compuesto } & Tiempo de retención & Porcentaje (\%) \\
\hline Ácido láurico & 10,350 & 0,55 \\
\hline Ácido mirístico & 15,432 & 0,54 \\
\hline Ácido palmítico & 20,439 & 13,24 \\
\hline Ácido linoleico & 24,452 & 60,48 \\
\hline Ácido oleico & 24,552 & 18,18 \\
\hline Ácido eláidico & 24,637 & 2,34 \\
\hline Ácido esteárico & 25,084 & 3,74 \\
\hline Ácido cis-11-eicosanoico & 28,837 & 0,42 \\
\hline Ácido eicosanoico & 29,388 & 0,35 \\
\hline Ácido docosanoico & 33,396 & 0,15 \\
\hline
\end{tabular}

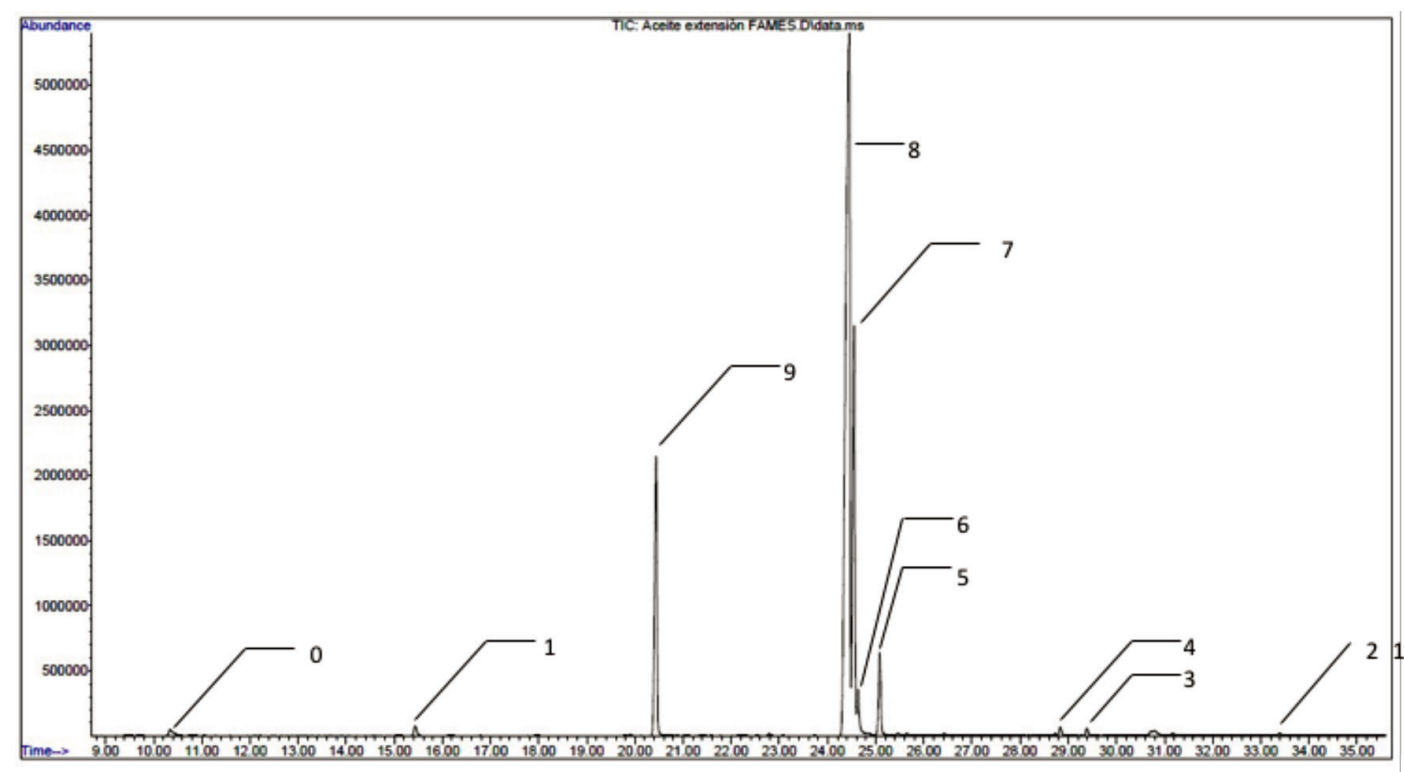

Figura 1. Perfil cromatográfico de ácidos grasos del aceite extraído: 1: ácido laúrico. 2: ácido mirístico. 3: ácido palmítico. 4: ácido linoleico. 5: ácido oleico. 6: ácido eláidico. 7: ácido esteárico. 8: ácido cis-11eicosanoico. 9: ácido eicosanoico. 10: ácido docosanoico.

\section{Determinación de compuestos fenólicos en el aceite}

Se realizó el análisis de compuestos fenólicos en el aceite mediante cromatografía gaseosa acoplada a masas, obteniendo como resultado la ausencia de este tipo de compuestos de acuerdo a la comparación con la biblioteca espectral NIST. Sin embargo, en este análisis se logró detectar la presencia de tocoferoles (terpenos de tipo fenólico). Las demás señales representativas de este cromatograma corresponden a los ácidos grasos propios del aceite. La Figura 2 muestra el cromatograma obtenido según la metodología utilizada y la Tabla 2 muestra los tocoferoles encontrados. 


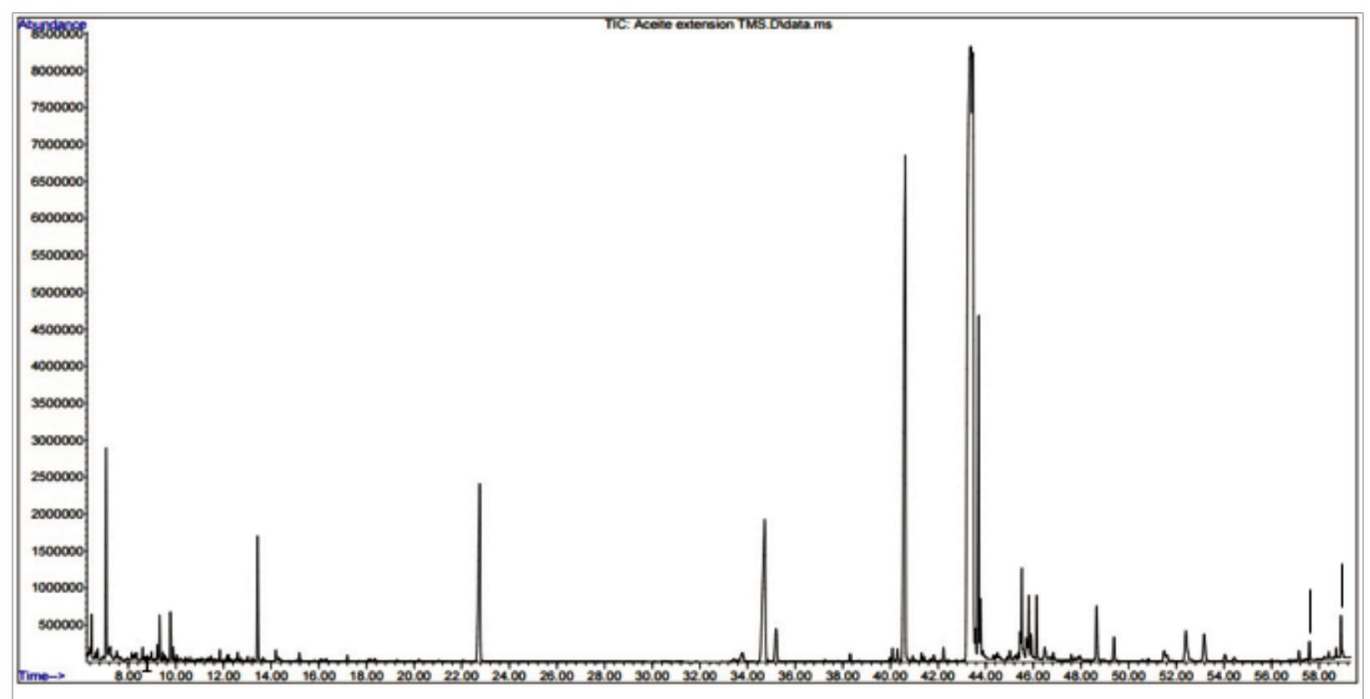

Figura 2. Perfil cromatográfico de trimetilsilil éster (TMS). 1: $\beta$-tocoferol. 2: $\alpha$-tocoferol

Tabla 2. Tocoferoles presentes en aceite de semillas de Ulex europaeus.

\begin{tabular}{l|c|c}
\hline \multicolumn{1}{c}{ Compuesto } & Tiempo de retención (min) & Porcentaje (\%) \\
\hline$\beta$-tocoferol & 57,59 & $1,02 \%$ \\
\hline$\alpha$-tocoferol & 58,91 & $0,65 \%$ \\
\hline
\end{tabular}

\section{Resultados y discusión}

Se empleó el método de extracción con hexano como solvente orgánico para solubilizar y aislar los ácidos grasos, el cual ha sido ampliamente utilizado para propósitos similares en otras plantas (Carrapiso \& García, 2000; Tian et al., 2013). De manera general, las características organolépticas y químicas de las fracciones lipídicas obtenidas a partir de las semillas de $U$. europaeus resultaron similares a lo informado por otros autores para la familia (Jobson \& Brynmor, 1964). El rendimiento promedio determinado $(7,33 \%)$ se encuentra en el promedio de los rendimientos reportados por otros autores para la familia (1,5 - $18 \%$ ) (Kirbride et al., 2003) según el método de extracción empleado. No obstante, los hallazgos en la presente investigación son preliminares por lo que se requiere estudios posteriores.

Se obtuvo un aceite con un 7,33\% de rendimiento promedio. El análisis de dicho aceite por cromatografía de gases (GC) acoplado a espectrometría de masas (MS), mostró la presencia de 10 tipos de ácidos grasos (Tabla 1). Los ácidos grasos mayoritarios fueron: Linoleico $60,48 \%$, Oleico con $18,18 \%$ y palmítico con 5,8\%. De manera general los resultados confirman los conseguidos por Jobson \& Brynmor (1964) quienes realizaron las descripciones de cantidad de lípidos, proteínas y fibra, sin discriminar los diferentes tipos de ácidos grasos que componía la fracción lipídica de la semilla.

El ácido graso más abundante en las semillas fue el linoleico $(60,48 \%)$. Este ácido es esencial para los humanos, debido a la carencia de las enzimas necesarias para asociar dobles enlaces en los átomos de carbono que están más allá del carbono 9 (Ronayne de Ferrer, 2000; Sanhueza et al., 2002; Galgani, 2004; Tapia, 2005; Ceron et al., 2012), su importancia radica fundamentalmente en que es un precursor de ácidos grasos de mayor longitud de cadena, entre ellos, el araquidónico que es un ácido 
graso esencial poli-insaturado de la serie omega-6, presente en membranas celulares, importante en la producción de prostaglandinas y otras interacciones de ácidos esenciales (Cerón et al., 2012). El ácido graso poli-insaturado linoleico y los demás ácidos grasos identificados en las semillas evaluadas, tienen diferentes usos industriales, en el caso del oleico, $18,18 \%$, es útil en la formulación cosmética y en mezclas con aceites minerales (Cerón et al., 2012), el palmítico, 13,24 \%, con frecuencia se utiliza como acidificante en emulsiones (Martini, 2005), y también, los ácidos grasos linoleico y oleico son compuestos utilizables como emolientes en productos cosméticos (Benaiges, 2008).

En los resultados conseguidos se puede apreciar la existencia de dos especies de tocoferoles en las muestras de las semillas evaluadas. Los Tocoferoles son el nombre genérico para una familia formada por 8 compuestos conformados por varios fenoles metilados con actividad de vitamina E (Burton \& Ingold, 1981). La forma más común es el tocoferol alfa y usualmente este compuesto se añade a suplementos vitamínicos y se utiliza también como antioxidante para la conservación de alimentos (Rigotti, 2007). Los tocoferoles son los antioxidantes más ampliamente distribuidos en la naturaleza y los más importantes que contienen naturalmente los aceites vegetales, su importancia radica en que ejercen su actividad antioxidante a concentraciones relativamente bajas que van desde $0,2 \%$ al 1\% de la fracción oleosa (Burton and Ingold, 1984; Schuler, 1990). El $\beta$-tocoferol (beta-tocoferol) es otra de las 4 formas naturales de tocoferol además de la alfa, tiene menor actividad antioxidante que el alfatocoferol debido a que carece de un grupo metilo en el esterocentro. En el estudio se reporta el $0,65 \%$ de la fracción oleosa de la semilla para $\bigotimes$-tocoferol contra un $1,02 \%$ del $\beta$-tocoferol. lo cual se confirma la actividad antioxidante para semillas de retamo espinoso debido a las concentraciones del grupo de tocoferoles en este trabajo.

\section{Conclusiones}

El Ulex europeaus -retamo espinoso- es una especie invasora en Colombia, en este caso se reporta su presencia en los cerros orientales de Bogotá. De acuerdo al perfil lipídico encontrado hay ácidos grasos con aplicaciones para la nutrición animal y la presencia de importantes antioxidantes -tocoferoles- en sus semillas. Teniendo en cuenta estos aspectos y que el retamo espinoso se considera como una especie invasora en el territorio colombiano, podría ser utilizado con fines industriales dado que, aunque se reporta poca cantidad oleosa en la semilla, la cantidad de semilla disponible es grande debido a su alta tasa de crecimiento y dispersión. Así entonces la utilización de la semilla cosechada de áreas invadidas, para ser usada con fines industriales podría contribuir como una estrategia eficaz y rentable para el manejo biológico de la especie. Sin embargo, se necesitan estudios sobre la factibilidad económica, análisis de costo beneficio, investigaciones sobre el impacto que tendría el uso de las semillas sobre las poblaciones de esta especie con el fin de medir la efectividad de esta medida y evaluaciones del riesgo de incorporar esta esta especie en cadenas de valor sobre el aumento de las áreas invadidas actualmente y su dispersión en nuevas regiones.

\section{Agradecimientos}

La información presentada en este artículo es resultado del proyecto de investigación: "Estudio de las características del retamo espinoso (Ulex eropaeus) localizado en los cerros orientales de la ciudad de Bogotá para su aprovechamiento como medida de minimización del impacto ambiental causado por su presencia", aprobado en convocatoria interna de la UNAD. 


\section{Literatura Citada}

1. Amaya, V. \& Rengifo, L. (2010). Efecto del retamo espinoso (Ulex europaeus) sobre las aves de borde en un bosque altoandino. Ornitología Colombiana, 10, 11-25.

2. Beltrán-G, H. \& Barrera-Cataño, J. (2014). Caracterización de invasiones de Ulex europaeus $L$. de diferentes edades como herramienta para la restauración ecológica de bosques altoandinos, Colombia. Biota Colombiana, 15(2), 3-26.

3. Benaiges, A. (2008). Aceite de rosa mosqueta: composición y aplicaciones dermocosméticas. Offarm, 27(6):94 - 97.

4. Bernal, R., Gradstein R. \& Celis, M. (2016). Catálogo de plantas y líquenes de Colombia. Bogotá, Colombia. Instituto de Ciencias Naturales, Universidad Nacional de Colombia. http://catalogoplantasdecolombia.unal. edu.co/es/

5. Burton, G. \& Ingold, K. (1981). Antioxidant activity of vitamin $\mathrm{E}$ and related chain-breaking phenolic antioxidants in vitro. J.Am. Chem. Soc, 103, 6472-6477.

6. Burton, G. \& Ingold, K. (1984). B-Carotene: an usual type of lípid antioxidants. Science, 224, 569-573.

7. Cárdenas-López, D., Castaño Arboleda, N. \& Cárdenas-Toro, J. (2010). Análisis de riesgo de especies de plantas introducidas para Colombia. En: Baptiste, M., Castaño, N., Cárdenas-López, D., Gutiérrez, F. Gil, D., Lasso, C. (Eds.). Análisis de riesgo y propuesta de categorización de especies introducidas para Colombia. Bogotá, Colombia. Instituto Alexander von Humboldt.

8. Carrapiso, A. \& García, C. (2000). Development in lipid analysis: some new extraction techniques and in situ transesterification. Lipids, 35(11), 1167-1176.

9. Cerón, A., Osorio, O. \& Hurtado, A. (2012). Identificación de ácidos grasos contenidos en los aceites extraídos a partir de semillas de tres diferentes especies de frutas. Acta Agron, 61 (2), 117-123.

10. Galgani, E. (2004). Evaluación de la situación de ácidos grasos esenciales y derivados de cadena larga en la dieta de lactantes menores de un año. Rev. chil. Nutr, 31(1), 154 - 160.

11. Genovesi, P. \& Shine, C. (2004). European strategy on invasive alien species. Strasbourg. Francia: Council of Europe Publishing, Nature and Environment. 68 p.

12. Guio, L., Solorza, J \& Leal, L. (2015). Restauración ecológica en plantaciones forestales de Eucalyptus globulus Labill y Acacia melanoxylon R. Br. Revista de Investigación Agraria y Ambiental, 6 (2), 145-156. Recuperado de: http://hemeroteca.unad.edu.co/index. php/riaa/article/view/1412/1737
13. Jobson, H. \& Brynmor, T. (1964). The composition of gorse (Ulex europaeus). Journal of the science of food and agriculture. 15(9), 652-656.

14. Kaufman, S. (2012). Guide to Identification and the impacts and control of North American species Sylvan. Estados Unidos: Stackpole Books. 517 p.

15. Kirbride, J., Gunn, C. \& Weitzman, A. (2003). Fruits and seeds of genera in the subfamily Faboideae (Fabaceae). Tech. Bull, 1890, 1-1208.

16. Marriott, P. \& Shellie, R. (2002). Principles and applications of comprehensive two-dimensional gas chromatography. Trends Anal. Chem, 21 (9-10), 573-583.

17. Martini, M. (2005). Introducción a la dermofarmacia y a la cosmetología. Zaragoza España: Acribia S.A. 300 p.

18. Rigotti, A. (2007). Absorption, transport, and tissue delivery of vitamin E. Mol. Aspects Med, 28(5-6), 423-436.

19. Rivera, D. \& Obón, C. (1995). The ethnopharmacology of Madeira and Porto Santo Islands, a review. J. Ethnopharmacol, 46, 73-93.

20. Ronayne de Ferrer, P. (2000). Importancia de los ácidos grasos poli-insaturados en la alimentación del lactante. Arch Argent Ped, 98, 231 - 238.

21. Sanhueza, J., Nieto, S. \& Valenzuela, A. (2002). Ácido Linoleico Conjugado: Un ácido graso con isomeria trans potencialmente beneficioso. Rev. chil. Nutr, 29(2), 98-105.

22. Schuler, P. (1990). Natural Antioxidants exploited commercially. In Food Antioxidants, Ámsterdam: El Sevier. $99 \mathrm{p}$.

23. Spínola, V., Llorent-Martínez, E., Gouveia-Figueirac, S. \& Castilhoa, P. (2016). Ulex europaeus: from noxious weed to source of valuable isoflavones and flavanones. Industrial Crops and Products, 90, 9-27.

24. Tapia, A. (2005). La suplementación con ácidos grasos omega-3 disminuye la agresividad, hostilidad y el comportamiento antisocial. Rev. chil. Nutr, 32(2), 95 - 101.

25. Tian, Y., Xu, Z., Zheng, B. \& Martin, L. (2013). Optimization of ultrasonic-assisted extraction of pomegranate (Punicagranatum L.) seed oil. Ultrason Sonochem, 20(1), 202-208.

26. Tighe-Neira, R., Leonelli-Cantergiani, G., MontalbaNavarro, R., Cavieres-Acuña, C. \& Morales-Ulloa, D. (2014). Caracterización de Compost a Base de Espinillo en Relación a la norma chilena n28801. Agron. Mesoam, 25(2), 347-355.
Conflicto de Intereses

Los autores declaran no tener ningún conflicto de intereses

Recibido: Febrero 15 de 2017 Aceptado: Abril 03 de 2017 